\title{
Microscopic approach to nucleon spectra in hypernuclear non-mesonic weak decay
}

\author{
E. Bauer ${ }^{\mathrm{a}, *}$, G. Garbarino ${ }^{\mathrm{b}}$ \\ a Departamento de Física, Universidad Nacional de La Plata and IFLP, CONICET C.C. 67, 1900 La Plata, Argentina \\ b Dipartimento di Fisica Teorica, Università di Torino, I-10125 Torino, Italy
}

\section{A R T I C L E I N F O}

\section{Article history:}

Received 25 November 2010

Received in revised form 15 February 2011

Accepted 3 March 2011

Available online 21 March 2011

Editor: W. Haxton

\section{Keywords:}

$\Lambda$-hypernuclei

Non-mesonic weak decay

Two-nucleon induced decay

FSI

\begin{abstract}
A B S T R A C T
A consistent microscopic diagrammatic approach is applied for the first time to the calculation of the nucleon emission spectra in the non-mesonic weak decay of $\Lambda$-hypernuclei. We adopt a nuclear matter formalism extended to finite nuclei via the local density approximation, a one-meson exchange weak transition potential and a Bonn nucleon-nucleon strong potential. Ground state correlations and final state interactions, at second order in the nucleon-nucleon interaction, are introduced on the same footing for all the isospin channels of one- and two-nucleon induced decays. Single and double-coincidence nucleon spectra are predicted for ${ }_{\Lambda}^{12} \mathrm{C}$ and compared with recent KEK and FINUDA data. The key role played by quantum interference terms allows us to improve the predictions obtained with intranuclear cascade codes. Discrepancies with data remain for proton emission.
\end{abstract}

(c) 2011 Elsevier B.V. All rights reserved.
Being the only source of information available on strangenesschanging baryon interactions, the non-mesonic weak decay of $\Lambda$-hypernuclei has attracted considerable interest and experienced great advances in the last years [1-3]. These hadronic weak interactions, whose determination requires the solution of complex many-body problems also involving strong interaction physics, are important inputs, for instance, when investigating the thermal evolution and the stability of compact stars [4]. The non-mesonic decay width, $\Gamma_{\mathrm{NM}}=\Gamma_{1}+\Gamma_{2}$, is built up from one- $(1 N)$ and twonucleon induced (2N) decays, $\Gamma_{1}=\Gamma_{n}+\Gamma_{p}$ and $\Gamma_{2}=\Gamma_{n n}+\Gamma_{n p}+$ $\Gamma_{p p}$, with the isospin components given by $\Gamma_{N}=\Gamma(\Lambda N \rightarrow n N)$ and $\Gamma_{N N^{\prime}}=\Gamma\left(\Lambda N N^{\prime} \rightarrow n N N^{\prime}\right)$, with $N, N^{\prime}=n$ or $p$.

As noted in [5], from a quantum-mechanical point of view the only observable in non-mesonic weak decay are the rate $\Gamma_{\mathrm{NM}}$ and the spectra of the emitted nucleons. Each one of the elementary non-mesonic decays occurs in the nuclear environment, thus final state interactions (FSI) can modify the quantum numbers of the nucleons produced in such weak processes and new, secondary nucleons are emitted as well: this prevents the measurement of any of the non-mesonic partial decay rates.

Although the long-standing puzzles on $\Gamma_{n} / \Gamma_{p}$ and the asymmetry parameter have been solved recently [2], a detailed knowledge of the non-mesonic decay mechanisms is still missing, as demon-

\footnotetext{
* Corresponding author.

E-mail address: bauer@fisica.unlp.edu.ar (E. Bauer).
}

strated by the persistent discrepancies between theory and experiment on the nucleon spectra [6].

In this Letter we present for the first time a consistent microscopic calculation of the nucleon emission spectra. A nuclear matter formalism is adopted and results for single and doublecoincidence nucleon spectra are reported for ${ }_{\Lambda}^{12} \mathrm{C}$ within the local density approximation. Previous investigations [7] demonstrated the negligible effect of ring and RPA microscopic contributions on nucleon spectra. Ground state correlations (GSC) and FSI contributions are introduced here at second order in the nucleonnucleon interaction for the whole set of $1 N$ and $2 N$ isospin decay channels. The weak transition potential $V^{\Lambda N \rightarrow N N}$ contains the exchange of the full set of mesons of the pseudoscalar $(\pi, \eta, K)$ and vector octets $\left(\rho, \omega, K^{*}\right)$, with strong coupling constants and cut-off parameters deduced from the Nijmegen soft-core interaction NSC97f [8]; for the nucleon-nucleon interaction $V^{N N \rightarrow N N}$ we adopt the Bonn potential (with the exchange of $\pi, \rho, \sigma$ and $\omega$ mesons) [9]. Being fully quantum-mechanical, the present approach is expected to produce more reliable results for FSI than those based on the (semi-classical) nucleon rescattering given by intranuclear cascade (INC) models [10,11].

The many-body terms we consider are represented by the inmedium $\Lambda$ self-energy Feynman diagrams of Fig. 1, where we limit to two- and three-nucleon emission. Diagrams $D$ and $E$ contribute to $1 N$ decays. By considering all the possible time-orderings of the Feynman diagrams at second order in $V^{N N \rightarrow N N}$, one obtains Goldstone diagram contributions to $2 N$ and FSI-induced decays as well as quantum interference terms (QIT). FSI Goldstone diagrams 
have at least one $V^{N N \rightarrow N N}$ acting after $V^{\Lambda N \rightarrow N N}$. QIT always involve a FSI and are of two kinds: FSI amplitudes interfere with both $1 N(1 N-F S I$ QIT) and $2 N$ decay amplitudes (2N-FSI QIT). We shall use the term 'plain FSI' to indicate a FSI which is not a QIT. For example, from the $p p$ Feynman diagram of Fig. 1 one obtains the Goldstone diagrams of Fig. 2: diagram (a) provides a plain FSI contribution when a cut on $3 p 2 h$ states is considered (this cut is associated to three-nucleon emission), while for cuts on $2 p 1 h$ states (two-nucleon emission) one has $1 \mathrm{~N}$-FSI QIT; (b) is a $2 \mathrm{~N}$ decay contribution, introduced by GSC; $\left(c_{1}\right)$ and $\left(c_{2}\right)$ are QIT with two possible final states, $2 p 1 h$, contributing to $1 N$-FSI QIT, and $3 p 2 h$, leaving to $2 N$-FSI QIT (incidentally, they are vanishing since $p_{i}=h_{i}$ and then one of the two requirements on the Fermi level, $p_{i}>k_{F}$ and $h_{i} \leqslant k_{F}$, cannot be fulfilled).

From this analysis it is clear that a single Goldstone diagram can contribute to a plain FSI and to a QIT, depending on the final physical state one considers: this point is important to have an un-
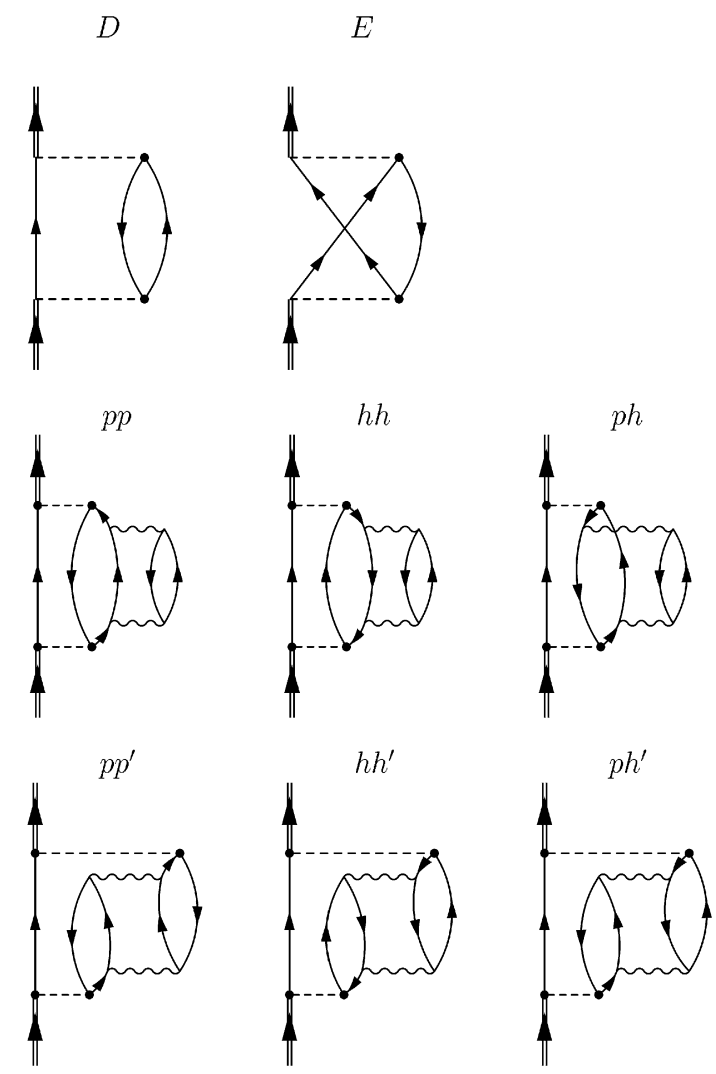

Fig. 1. The set of Feynman diagrams considered in this work for the in-medium $\Lambda$ self-energy. $D$ and $E$ are the direct and exchange terms of the $1 \mathrm{~N}$ decay channel, the remaining ones contribute to $2 \mathrm{~N}$ and FSI-induced decays. The dashed and wavy lines stand for the weak and strong potentials, $V^{\Lambda N \rightarrow N N}$ and $V^{N N \rightarrow N N}$, respectively.

(a)

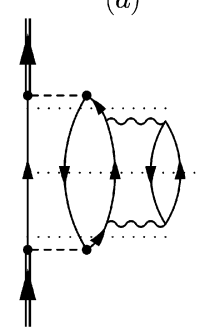

(b)

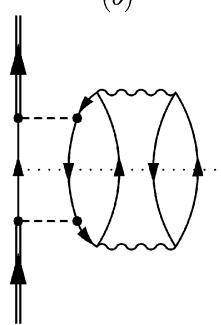

ambiguous interpretation of the QIT. A cut in a Goldstone diagram that leads to two symmetric pieces is interpreted as the square of a transition amplitude: a non-QIT which contains the interaction $V^{N N \rightarrow N N}$ can represent either a $2 N$ decay or a plain FSI-induced decay. At variance, when the cut divides the diagram into two different amplitudes, one has a QIT. $2 \mathrm{~N}$ and plain FSI contributions are positive-definite, while QIT can be either positive or negative. This trivial fact has important consequences that will be discussed later.

Regardless of the set of many-body contributions one considers, the Goldstone diagram technique allows us to write the total number of nucleons and nucleon pairs emitted in the non-mesonic decay as follows [7]:

$$
\begin{aligned}
& N_{n}=2 \bar{\Gamma}_{n}+\bar{\Gamma}_{p}+3 \bar{\Gamma}_{n n}+2 \bar{\Gamma}_{n p}+\bar{\Gamma}_{p p}+\sum_{i, f} N_{f(n)} \bar{\Gamma}_{i, f} \\
& N_{p}=\bar{\Gamma}_{p}+\bar{\Gamma}_{n p}+2 \bar{\Gamma}_{p p}+\sum_{i, f} N_{f(p)} \bar{\Gamma}_{i, f} \\
& N_{n n}=\bar{\Gamma}_{n}+3 \bar{\Gamma}_{n n}+\bar{\Gamma}_{n p}+\sum_{i, f} N_{f(n n)} \bar{\Gamma}_{i, f} \\
& N_{n p}=\bar{\Gamma}_{p}+2 \bar{\Gamma}_{n p}+2 \bar{\Gamma}_{p p}+\sum_{i, f} N_{f(n p)} \bar{\Gamma}_{i, f} \\
& N_{p p}=\bar{\Gamma}_{p p}+\sum_{i, f} N_{f(p p)} \bar{\Gamma}_{i, f}
\end{aligned}
$$

where a normalization per non-mesonic decay is used $(\bar{\Gamma} \equiv$ $\left.\Gamma / \Gamma_{\mathrm{NM}}\right)$. Single and double coincidence nucleon spectra are obtained by constraining the evaluation of each $\bar{\Gamma}$ to certain intervals in energy, opening angle, etc. The $\bar{\Gamma}_{N}$ 's $\left(\bar{\Gamma}_{N N^{\prime}}\right.$ 's) are the $1 N(2 N)$ decay rates, while the remaining terms containing the functions $\bar{\Gamma}_{i, f}$ represent FSI Goldstone diagrams (for instance, diagrams (a), $\left(c_{1}\right)$ and ( $\left.c_{2}\right)$ of Fig. 2). The index $i$ in $\bar{\Gamma}_{i, f}$ is used to label the FSI Goldstone diagrams obtained from the Feynman diagrams of Fig. 1 at second order in $V^{N N \rightarrow N N}$, while $f$ denotes the final physical states of the Goldstone diagram and in the present case can take the values $f=n N$ (cut on $2 p 1 h$ states) and $n N N^{\prime}$ (cut on $3 p 2 h$ states). Finally, $N_{f(N)}\left(N_{f\left(N N^{\prime}\right)}\right)$ is the number of nucleons of the type $N$ (of $N N^{\prime}$ pairs) contained in the multinucleon state $f$.

Note that Eqs. (1) contain five observables, $N_{N}$ and $N_{N N^{\prime}}$, and five unobservable decay widths, $\Gamma_{N}$ and $\Gamma_{N N^{\prime}}$. The unobservable character of the decay widths is due to the presence of FSI, which we know to be important. Therefore, one cannot invert the above relations to obtain the decay widths in terms of the observed spectra. The experimental values of the partial widths can be obtained from the measured spectra only if a model is used for deconvoluting the FSI effects.

To illustrate an important property of FSI, let us consider the diagram of Fig. 2(a) (we assign to it the index $i=1$ ) with $f=n N N^{\prime}$ (cut on $3 p 2 h$ states): it is represented by the function $\bar{\Gamma}_{1, n N N^{\prime}}$,

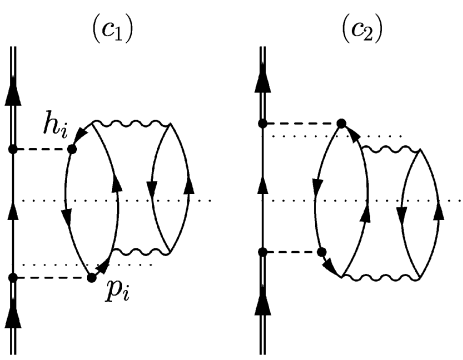

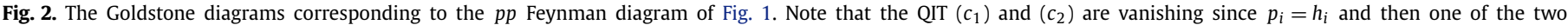
requirements on the Fermi level, $p_{i}>k_{F}$ and $h_{i} \leqslant k_{F}$, cannot be fulfilled. See text for details. 
which is a plain FSI-induced contribution. The digram in Fig. 2(a) can be cut also on $2 p 1 h$ states: in this case it contributes to the $1 N$-FSI QIT $\bar{\Gamma}_{1, n N}$. From sum rule considerations on Goldstone diagrams [12] it follows that

$$
\sum_{N=n, p} \bar{\Gamma}_{1, n N}+\sum_{N, N^{\prime}=n, p} \bar{\Gamma}_{1, n N N^{\prime}}=0 .
$$

In general, each FSI $\Lambda$ self-energy diagram can in no way contribute to the total non-mesonic rate:

$$
\begin{aligned}
& \sum_{N=n, p} \bar{\Gamma}_{i, n N}+\sum_{N, N^{\prime}=n, p} \bar{\Gamma}_{i, n N N^{\prime}} \\
& \quad+\sum_{N, N^{\prime}, N^{\prime \prime}=n, p} \bar{\Gamma}_{i, n N N^{\prime} N^{\prime \prime}}+\cdots=0,
\end{aligned}
$$

for any value of $i$, while in principle it can affect the $1 N, 2 N$, etc., decay rates separately (see however the discussion of [5] on the opportunity not to include these FSI terms in the definition of the unobservable partial rates). Note that the above sum rule is valid for the nucleon spectra integrated over the whole energy range for all the nucleons in the state $f$; the cancelation between the $3 p 2 h$ and $2 p 1 h$ contributions of each Goldstone diagram does not occur at a given value of the energy of one of the nucleons contained in $f$. Note also that the numbers $N_{f(N)}$ and $N_{f\left(N N^{\prime}\right)}$ prevent the above cancelation to occur for the total numbers in Eqs. (1) too. Thus, QIT diagrams surely play a role in nucleon spectra.

Let us emphasize some consequences of the sum rule condition in Eq. (3). First, while $\Gamma_{n}$ and $\Gamma_{p}$ are not observable, the total decay width $\Gamma_{\mathrm{NM}}$ (which is unaffected by FSI) is an observable. Second, it is obvious that the cancellation in Eqs. (2) and (3) occurs because some terms are negative: these terms are necessarily QIT. It is well known that FSI are important; in addition the second property above tells us that the magnitude of QIT is the same as the one of plain FSI in the evaluation of $\Gamma_{\mathrm{NM}}$. This proportion can change in the evaluation of the spectra due to the presence of the numbers $N_{f(N)}$ and $N_{f\left(N N^{\prime}\right)}$, but in no way QIT can be neglected: without QIT, the emission spectra would be overestimated. It is important to stress that this discussion refers to a quantum mechanical treatment only.

Before discussing our numerical results, it should be mentioned that the values of $\bar{\Gamma}_{N}, \bar{\Gamma}_{N N^{\prime}}$ and $\bar{\Gamma}_{i, f}$ are obtained from the corresponding $\Lambda$ self-energy Goldstone diagrams. Explicit expressions for diagrams $D$ and $E$ in Fig. 1 are found in [13]. For the formal derivation of the decay rates from the $p p, p h, h h, p p^{\prime}, p h^{\prime}$ and $h h^{\prime}$ Feynman diagrams in the same figure, we proceed as follows. The evaluation of the $p p$, $p h$ and $h h$ Goldstone diagrams contributing to $2 N$ decays can be found in [14] ( $p p$ in the main text, the other two in the appendix). The expressions for the same timeorderings for $p p^{\prime}, p h^{\prime}$ and $h h^{\prime}$ diagrams are shown in the appendix of [15]. The remaining time-ordering formulas for $p p, p h, h h, p p^{\prime}$, $p h^{\prime}$ and $h h^{\prime}$ can be obtained from the just mentioned ones after some work: in any case, the main ingredients are already present in the mentioned works.

As a starting point for the numerical analysis, in Table 1 we show the decay rates obtained for ${ }_{\Lambda}^{12} \mathrm{C}$. We also predict that $\Gamma_{n p} / \Gamma_{2}=0.84, \Gamma_{p p} / \Gamma_{2}=0.12$ and $\Gamma_{n n} / \Gamma_{2}=0.04$. The agreement with the recent KEK and FINUDA data is quite satisfactory.

We turn now to our main concern in this Letter, which is the study of the emission spectra. The obtained emission spectra depend mainly on the phase space and the nucleon-nucleon strong potential. The fact that these spectra are normalized per non-mesonic weak decay makes the dependence on the weak interaction to be very small. In the present contribution we have employed the weak and strong interaction models used in [5].
Table 1

The non-mesonic decay widths predicted for ${ }_{\Lambda}^{12} \mathrm{C}$ (in units of the free decay rate). The most recent data, from KEK-E508 [16] and FINUDA [17], are also given.

\begin{tabular}{llll}
\hline Decay rate & Our & KEK-E508 & FINUDA \\
\hline$\Gamma_{n}$ & 0.15 & $0.23 \pm 0.08$ & \\
$\Gamma_{p}$ & 0.47 & $0.45 \pm 0.10$ & \\
$\Gamma_{1}$ & 0.62 & $0.68 \pm 0.13$ & \\
$\Gamma_{2}$ & 0.36 & $0.27 \pm 0.13$ & \\
$\Gamma_{\mathrm{NM}}$ & 0.99 & $0.95 \pm 0.04$ & \\
$\Gamma_{n} / \Gamma_{p}$ & 0.33 & $0.51 \pm 0.13 \pm 0.05$ & $0.24 \pm 0.10$ \\
$\Gamma_{2} / \Gamma_{\mathrm{NM}}$ & 0.37 & $0.29 \pm 0.13$ & \\
\hline
\end{tabular}

These parameterizations turned out to reproduce the full set of decay widths for carbon hypernuclei determined recently at KEK [16]. Note that the use of the same strong interaction model for both GSC and FSI not only provides internal consistency to the model, but gives us a criterion to select the strong interaction potential, since modifications of this potential leads to testable changes in both $\Gamma_{2} / \Gamma_{\mathrm{NM}}$ and the nucleon spectra.

In Fig. 3 we show the neutron and proton kinetic energy spectra for the non-mesonic decay of ${ }_{\Lambda}^{12} \mathrm{C}$. The dashed curves are the distributions of the $1 \mathrm{~N}$ decay nucleons (normalized per $1 \mathrm{~N}$ decay): as expected, they show a maximum at half of the $Q$-value for ${ }_{\Lambda}^{12} \mathrm{C}$ non-mesonic decay and a bell-type shape due to the nucleon Fermi motion and the $\Lambda$ momentum distribution in the hypernucleus. The inclusion of $2 \mathrm{~N}$ and FSI-induced decay processes provides the results given by the continuous lines (normalized per non-mesonic decay) and leads to a reduction of the nucleon average energy, thus filling the low-energy part of the spectrum and emptying the high-energy region. This outcome is explained as follows. The dominant contribution to FSI is provided by Fig. 2(a): according to phase space arguments, when a cut on $3 p 2 h$ states is considered one obtains a distribution that decreases monotonically with $T_{N}$, while a cut on $2 p 1 h$ states provides a bell-shaped, negative QIT peaked at $T_{N} \simeq 70 \mathrm{MeV}$. Another important contribution is that of Fig. 2(b), which admits $3 p 2 h$ final states only: it produces positive and monotonically decreasing nucleon distributions with the same order of magnitude of the $3 p 2 h$ term of Fig. 2(a). While we reproduce fairly well the KEK neutron spectra, a rather strong overestimation is found of the KEK-E369 and FINUDA proton distributions. Our proton spectrum is instead closer to the old BNL-KEK data.

Also from Fig. 3 we see a certain dispersion among the various experimental proton spectra (for instance, FINUDA shows a peaking structure at $T_{p} \simeq 80 \mathrm{MeV}$ which is not seen in the other data nor in this and in previous calculations). Compared with the nucleon spectra obtained with the INC model for FSI in [6] (dotdashed curves), we obtain here similar results for $T_{N} \gtrsim 40 \mathrm{MeV}$; the distributions of [6] for smaller $T_{N}$ increase well beyond the predictions of the present work, which are more reliable than the ones based on the INC in this energy regime. Given the obvious differences between the present microscopic approach and the INC rescattering model, the previous agreement is a remarkable result. The QIT of the present approach replace the nucleon rescattering present in the INC, whose effect is to reduce the energy of the nucleons propagating through the residual nucleus.

The opening angle distributions of $n n$ and $n p$ pairs are reported in Fig. 4. To adhere to the KEK data, the predictions of the full model are obtained for a $30 \mathrm{MeV}$ nucleon kinetic energy threshold $T_{N}^{\text {th }}$. The distributions from the $1 N$ decay (dashed curves) are strongly peaked at $\theta_{N N^{\prime}}=180^{\circ}$. The QIT again have a crucial effect: they considerably reduce the back-to-back contribution, thanks mainly to diagram in Fig. 2(a) with $2 p 1 \mathrm{~h}$ final states; the non back-to-back region is strongly populated mainly by the 

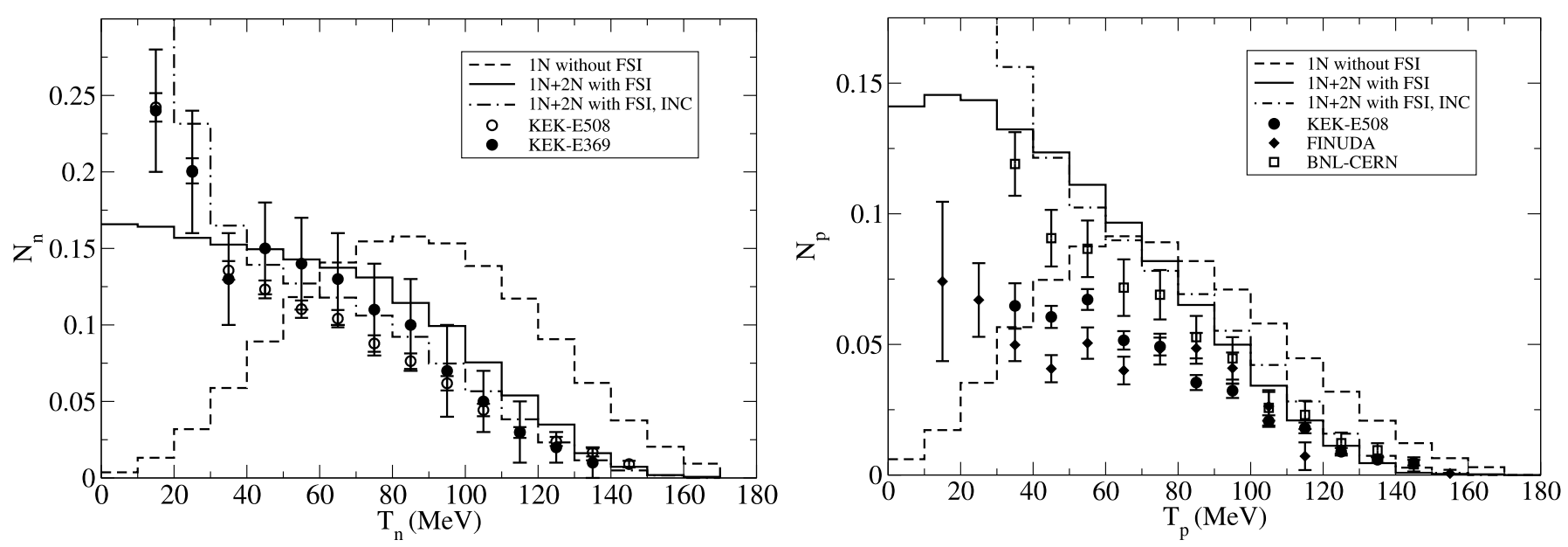

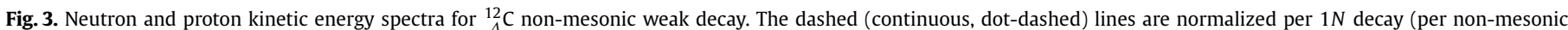
decay). Experimental data are from KEK-E369 [18], KEK-E508 [19], FINUDA [17] and BNL-CERN [20].
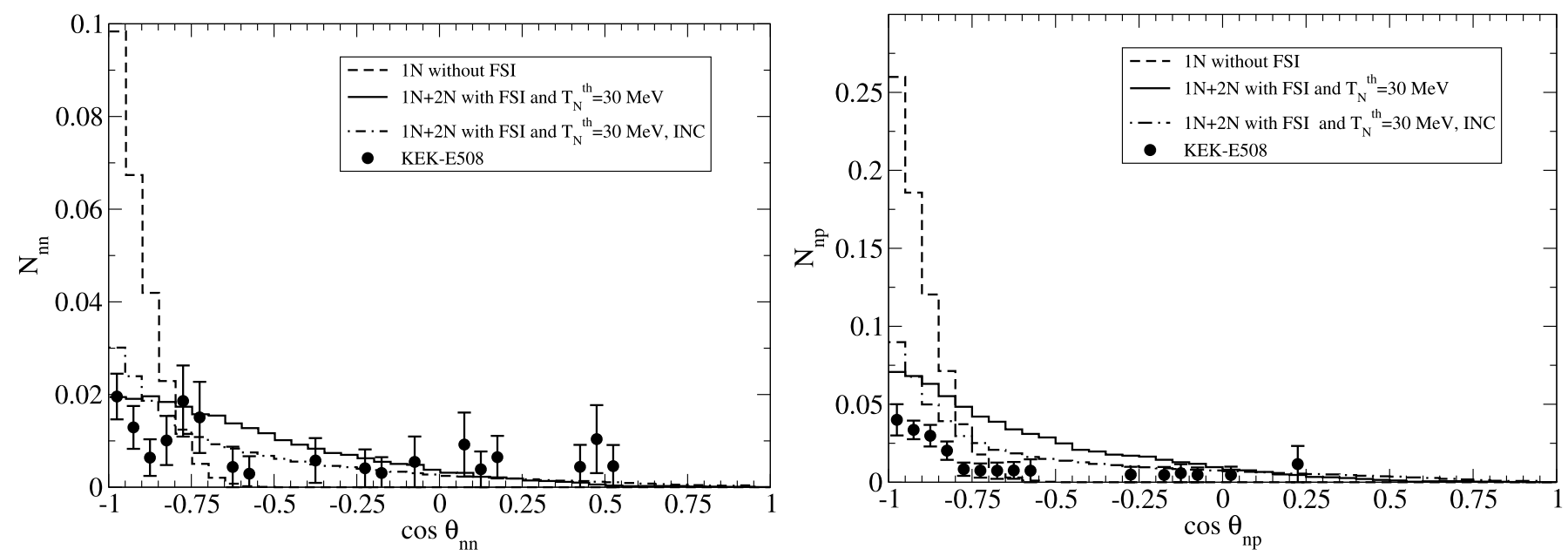

Fig. 4. Opening angle distribution of $n n$ and $n p$ pairs. Normalization is as in Fig. 3. Data are from KEK-E508 [21].

$3 p 2 h$ contributions of diagrams in Figs. 2(a) and 2(b) (see continuous curves). The final results for the angular correlations turn out to be somewhat less back-to-back peaked than what found in [6] (dot-dashed curves), and, as in that work, are very sensitive to the value adopted for $T_{N}^{\text {th }}$. The agreement with KEK-E508 data is rather good for the $n n$ spectrum, while for $n p$ pairs a significant overestimation is obtained. Note that we reproduce for the first time the experimental $n n$ opening angle spectrum: it is the quantummechanical nature of our scheme, i.e., the relevance of QIT, which brings to this achievement. Our overestimation of the KEK-E508 $\mathrm{np}$ distribution is compatible with the overestimation of the proton spectrum obtained in the same experiment (see Fig. 3).

In Fig. 5 we give the two-nucleon momentum correlation spectra, i.e., the $n n$ and $n p$ distributions as a function of the momentum sum $p_{N N^{\prime}} \equiv\left|\vec{p}_{N}+\vec{p}_{N^{\prime}}\right|$ of two of the outgoing nucleons. The dashed lines correspond to the $1 N$ decay, while the continuous curves show the full result, with $1 \mathrm{~N}, 2 \mathrm{~N}$ and FSI included together with a nucleon kinetic energy threshold $T_{N}^{\text {th }}=30 \mathrm{MeV}$, as in the data also shown in the figures. As noted in Ref. [16], the minimum in both the $n n$ and $n p$ KEK-E508 distributions is mainly due to the low statistics and detection efficiency for events with $p_{N N^{\prime}} \gtrsim 350 \mathrm{MeV} / c$ (the KEK detector geometry being optimized for back-to-back coincidence events); indeed, such dip structure has not been found in our calculation, which overestimates the data for large correlation momenta (especially for $N_{n p}$, consistently with the spectra discussed so far).

The distributions of Fig. 5 at low momentum sum (say below $400 \mathrm{MeV} / \mathrm{c}$ ) are mainly due to $1 \mathrm{~N}$ decays (which are strongly backto-back correlated), while for higher momenta the contribution of $2 \mathrm{~N}$ and FSI-induced decays is dominant (and produces less backto-back correlated pairs). This behavior is confirmed by the momentum correlation of the sum $N_{n n}+N_{n p}$ shown in Fig. 6 for the opening angle regions with $\cos \theta_{N N^{\prime}}<-0.7$ and $\cos \theta_{N N^{\prime}}>-0.7$.

In Table 2 we show results for the coincidence numbers $N_{n n}$, $N_{n p}$ and $N_{p p}$ obtained for $T_{N}^{\text {th }}=30 \mathrm{MeV}$ and two angular regions, $\cos \theta_{N N^{\prime}}<-0.7$ and $\cos \theta_{N N^{\prime}}>-0.7$. Previous predictions of finite nucleus [11] and nuclear matter approaches [6], both based on the INC, and experimental data are also given. Again, a remarkable result is the fact that the present predictions are very similar to the ones of [6], while one notes less agreement with [11], mainly due to the different models adopted to describe $2 \mathrm{~N}$ decays. Comparison with data shows an overestimation of $N_{n p}$ and $N_{p p}$ but rather good results for $N_{n n}$. This confirms a systematic overestimation of the proton emission reported by KEK-E508.

Summarizing, a microscopic approach including GSC and FSI on the same footing is used to evaluate the nucleon emission spectra in non-mesonic weak decay of hypernuclei. Within our microscopic model, QIT play a key role: in the single-nucleon emission spec- 

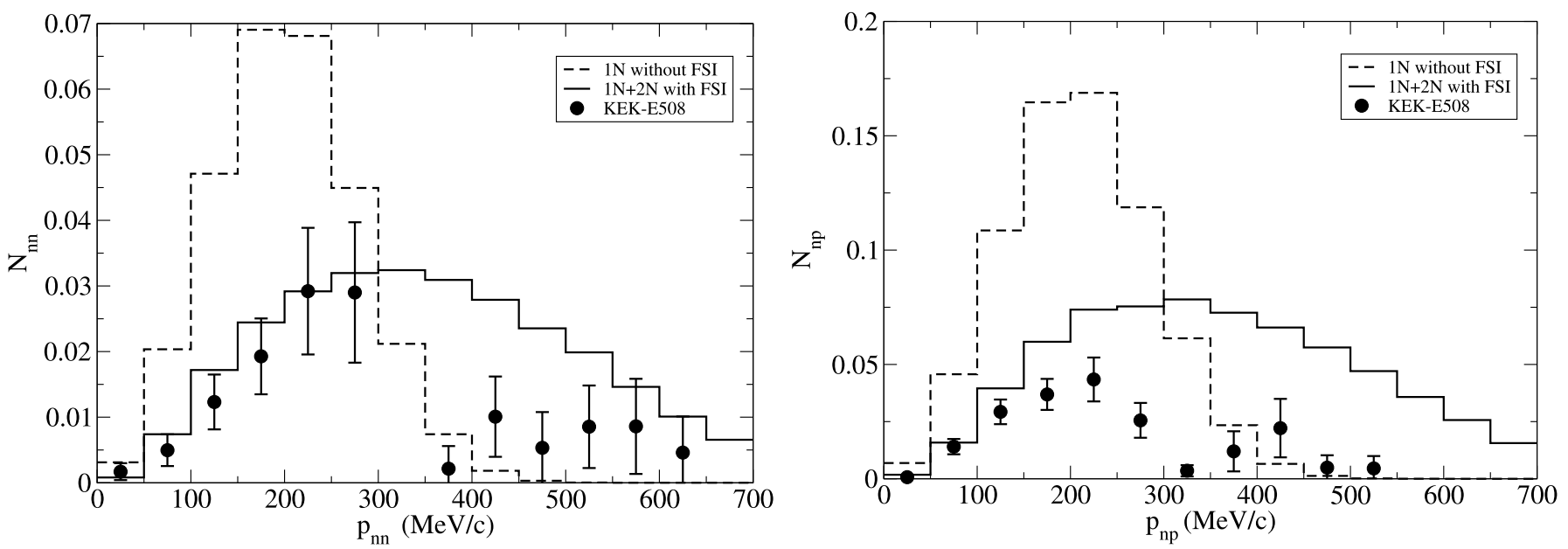

Fig. 5. Momentum correlation spectra of $n n$ and $n p$ pairs, with $p_{N N^{\prime}} \equiv\left|\vec{p}_{N}+\vec{p}_{N^{\prime}}\right|$. Normalization is as in Fig. 3. Data are from KEK-E508 [16].

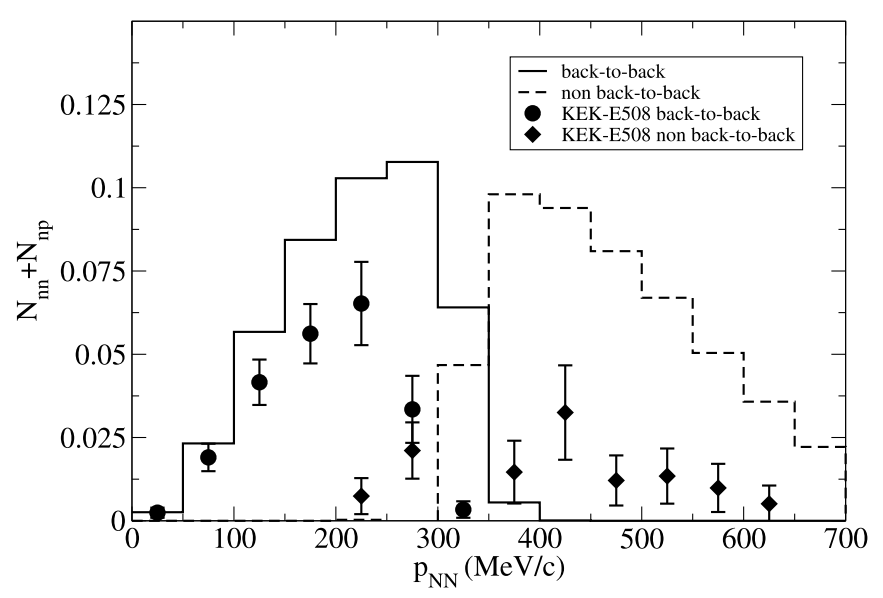

Fig. 6. Momentum correlation spectra for the sum of the $n n$ and $n p$ pair numbers for the back-to-back ( $\cos \theta_{N N}<-0.7$, continuous line) and non-back-to-back kinematics $\left(\cos \theta_{N N}>-0.7\right.$, dashed line). Normalization is per non-mesonic weak decay. Data are from KEK-E508 [22].

\section{Table 2}

The nucleon coincidence numbers are given for $T_{N}^{\text {th }}=30 \mathrm{MeV}$ and the angular regions with $\cos \theta_{N N^{\prime}}<-0.7$ and $\cos \theta_{N N^{\prime}}>-0.7$ (in parenthesis). Data are from KEK-E508 [21].

\begin{tabular}{llll}
\hline & $N_{n n}$ & $N_{n p}$ & $N_{p p}$ \\
\hline This work & $0.11(0.13)$ & $0.35(0.31)$ & $0.04(0.05)$ \\
INC [11] & $0.15(0.18)$ & $0.35(0.52)$ & $0.08(0.27)$ \\
INC [6] & $0.11(0.10)$ & $0.30(0.25)$ & $0.05(0.07)$ \\
KEK-E508 & $0.083 \pm 0.014$ & $0.138 \pm 0.014$ & $0.005 \pm 0.002$ \\
& $(0.083 \pm 0.020)$ & $(0.060 \pm 0.018)$ & \\
\hline
\end{tabular}

tra they are responsible for moving intensity from the high-energy region to the low-energy region, while in the opening angle distribution of nucleon pairs the strong reduction of the back-to-back peak is entirely due to QIT. Discrepancies with experiment remain, but are relegated to spectra involving protons. Further work is in order to understand such a disagreement. A forthcoming coincidence experiment at J-PARC [23] will allow a measurement of the nucleon spectra and a determination of $\Gamma_{n}, \Gamma_{p}$ and $\Gamma_{2}$ for ${ }_{1}^{12} \mathrm{C}$ with improved accuracy. Moreover, our microscopic approach is particularly suitable for the inclusion of new decay mechanisms introduced by fermion antisymmetrization and the $\Delta(1232)$-resonance. These contributions deserve consideration.

\section{References}

[1] W.M. Alberico, G. Garbarino, Phys. Rep. 369 (2002) 1.

[2] G. Garbarino, Nucl. Phys. A 835 (2010) 144.

[3] H. Outa, Hadron Physics, in: T. Bressani, A. Filippi, U. Wiedner (Eds.), Proceedings of the International School of Physics "Enrico Fermi", Course CLVIII, Varenna (Italy), June 22-July 2, 2004, IOS Press, Amsterdam, 2005, p. 219.

[4] J. Schaffner-Bielich, Nucl. Phys. A 835 (2010) 279.

[5] E. Bauer, G. Garbarino, Phys. Rev. C 81 (2010) 064315.

[6] E. Bauer, G. Garbarino, A. Parreño, A. Ramos, Nucl. Phys. A 836 (2010) 199.

[7] E. Bauer, Nucl. Phys. A 781 (2007) 424; E. Bauer, Nucl. Phys. A 796 (2007) 11.

[8] V.G.J. Stoks, Th.A. Rijken, Phys. Rev. C 59 (1999) 3009; Th.A. Rijken, V.G.J. Stoks, Y. Yamamoto, Phys. Rev. C 59 (1999) 21.

[9] R. Machleidt, K. Holinde, Ch. Elster, Phys. Rep. 149 (1987) 1.

[10] A. Ramos, M.J. Vicente-Vacas, E. Oset, Phys. Rev. C 55 (1997) 735; A. Ramos, M.J. Vicente-Vacas, E. Oset, Phys. Rev. C 66 (2002) 039903, Erratum.

[11] G. Garbarino, A. Parreño, A. Ramos, Phys. Rev. Lett. 91 (2003) 112501; G. Garbarino, A. Parreño, A. Ramos, Phys. Rev. C 69 (2004) 054603.

[12] W.M. Alberico, A. De Pace, A. Drago, A. Molinari, Riv. Nuovo Cimento 14 (5) (1991) 1.

[13] E. Bauer, F. Krmpotić, Nucl. Phys. A 717 (2003) 217.

[14] E. Bauer, F. Krmpotić, Nucl. Phys. A 739 (2004) 109.

[15] E. Bauer, Nucl. Phys. A 818 (2009) 174.

[16] M. Kim, et al., Phys. Rev. Lett. 103 (2009) 182502.

[17] M. Agnello, et al., Phys. Lett. B 685 (2010) 247.

[18] J.H. Kim, et al., Phys. Rev. C 68 (2003) 065201.

[19] S. Okada, et al., Phys. Lett. B 597 (2004) 249.

[20] A. Montwill, et al., Nucl. Phys. A 234 (1974) 413.

[21] M.J. Kim, et al., Phys. Lett. B 641 (2006) 28.

[22] H. Bhang, M. Kim, private communication.

[23] M. Kim, et al., Nucl. Phys. A 835 (2010) 434;

$\mathrm{H}$. Bhang, et al., Coincidence measurement of the weak decay of ${ }_{\Lambda}^{12} \mathrm{C}$ and the three-body weak interaction process, Letter of intent for an experiment (E18) at J-PARC, 2006. 